\title{
A99-36580
}

\section{CONTROL DESIGN FOR THE ACTEX FLIGHT EXPERIMENT USING THE ROBUST, FIXED-STRUCTURE TOOLBOX}

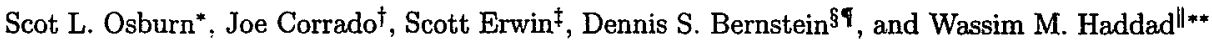

\begin{abstract}
In this paper, we implement fixed-structure controller synthesis methods to the ACTEX flight experiment. We show that the decentralized static output feedback formulation of fixed-structure controller synthesis can directly account for the control-structure constraints of the ACTEX flight experiment. Finally, we show that the ACTEX controller structure can be written as a decentralized static output feedback problem and obtain feedback controllers for suppressing broadband disturbances.
\end{abstract}

\section{Introduction}

The ACTEX flight experiment provides a unique opportunity for users to implement and test controllers on a spacebased platform. In this regard, the hardware environment has several features that must be accounted for in specifying control algorithms.

First, the feedback control algorithms that can be implemented on ACTEX are fixed gain, and thus adaptive controllers cannot be used. Furthermore, these fixed-gain controllers are analog, which avoids sampling effects. Finally, the implementable analog controllers have a prespecified structure in which only filter gains and natural frequencies can be modified. Since this constraint does not permit implementation of dynamic compensators of arbitrary structure, standard LQG and $\mathcal{H}_{\infty}$ methods cannot be applied.

In this paper we apply fixed-structure controller synthesis methods to the ACTEX flight experiment. Fixed-structure methods have been extensivcly developed in $\mathcal{H}_{2}$ and $\mathcal{H}_{2}$ $/ \mathcal{H}_{\infty}$ settings in continuous and discrete time [1]-[8]. In its

\footnotetext{
*Aerospace Engineering Department, The University of Michigan, Ann Arbor, MI, U.S.A.

'School of Aerospace Engineering, Georgia Institute of Technology, Atlanta, GA, U.S.A.

${ }^{\ddagger}$ Air Force Research Laboratory, Space Vehicles Directorate, Kirtland AFB, NM, U.S.A.

${ }^{\S}$ Professor, Aerospace Engineering Department, The University of Michigan, Ann Arbor, MI, U.S.A.

TThis research was supported in part by the Air Force Office of Scientific Research under grant F49620-98-1-0037.

llProfessor, School of Aerospace Engineering, Georgia Institute of Technology, Atlanta, GA, U.S.A.

**This research was supported in part by the Air Force Office of Scientific Research under grant F49620-96-I-0125.

${ }^{1}$ Copyright (C) 1999 The Anerican Institute of Atronautics and Astronautics Inc. All rights reserved.
}

most general form, fixed-structure synthesis has been developed for affinely parameterized closed-loop dynamics which corresponds to a decentralized static output formulation [6].

In the present paper we show that the decentralized static output feedback formulation of fixed-structure controller synthesis can directly account for the control-structure constraints of the ACTEX flight experiment. Specifically, we show that the ACTEX controller structure can be written as a decentralized static output feedback problem. Having done this, we then proceed to apply the techniques of [6] to obtain feedback controllers for suppressing broadband disturbances.

\section{Structural Dynamics Modeling of the ACTEX Flight Experiment}

The ACTEX flight experiment consists of a plate connected to a satellite by 3 struts. Each strut is equipped with its own control piezo-actuator as well as a colocated and nearly colocated sensor. A disturbance can be introduced to the experimental package through each of the 3 control actuators, or through a disturbance actuator on the plate. In addition, each of the 3 control actuators has an independent decentralized analog controller.

The dynamics of the ACTEX experiment can be represented by the continuous-time system

$$
\begin{aligned}
& \dot{x}(t)=A x(t)+B u(t)+D_{1} w(t), \\
& y(t)=C x(t)+D u(t)+D_{2} w(t),
\end{aligned}
$$

where $x \in \mathcal{R}^{n}, u \in \mathcal{R}^{m}, y \in \mathcal{R}^{l}$, and $w \in \mathcal{R}^{d}$ are the state, input, measurement, and disturbance, respectively. The disturbance $w$ is a standard zero-mean white noise process. The performance variables are given by

$$
z(t)=E_{1} x(t)+E_{2} u(t)+D_{0} w(t)
$$

Several experiments have been run on the ACTEX package, with telemetry returned for identification purposes. Measurements were taken from onboard accelerometers, sensor inputs, and actuator outputs, sampled at $4 \mathrm{kHz}$. Using this data, identification was performed on the plant from the strut 1 actuator to the strut 1 nearly-colocated sensor. For frequencies below $200 \mathrm{~Hz}$, these dynamics can be repre- 
sented by (1)-(3) where

$$
\begin{aligned}
& A=\left[\begin{array}{cccc}
-1.56 & 77.98 & 0 & 0 \\
-77.98 & -1.56 & 0 & 0 \\
0 & 0 & -0.25 & 24.99 \\
0 & 0 & -24.99 & -0.25
\end{array}\right], \quad B=\left[\begin{array}{r}
-1.10 \\
-0.21 \\
-0.11 \\
0.03
\end{array}\right] \\
& C=\left[\begin{array}{llll}
-0.15 & 0.70 & 1.83 & 6.88
\end{array}\right], \cdots D=1, \\
& E_{1}=\left[\begin{array}{cccc}
-0.15 & 0.70 & 1.83 & 6.88 \\
0 & 0 & 0 & 0
\end{array}\right], \quad E_{2}=\alpha\left[\begin{array}{l}
0 \\
1
\end{array}\right], \\
& D_{1}=\left[\begin{array}{rr}
-1.10 & 0 \\
-0.21 & 0 \\
-0.11 & 0 \\
0.03 & 0
\end{array}\right], \quad D_{2}=\left[\begin{array}{ll}
0 & 0.1
\end{array}\right], \quad D_{0}=\left[\begin{array}{ll}
0 & 0 \\
0 & 0
\end{array}\right] \text {. }
\end{aligned}
$$

In this paper, we will consider this SISO problem, with input at the strut 1 sensor, and output at the strut 1 actuator. In the next section, : we review the decentralized static output feedback framework, and express the ACTEX model in this representation.

\section{Review of Fixed-Structure Synthesis}

In this section we use the fixed-structure control framework given in [6] to transform the $\mathcal{H}_{2}$-optimal ACTEX control problem to a decentralized static output feedback setting. Consider the 4-vector input, 4-vector output decentralized

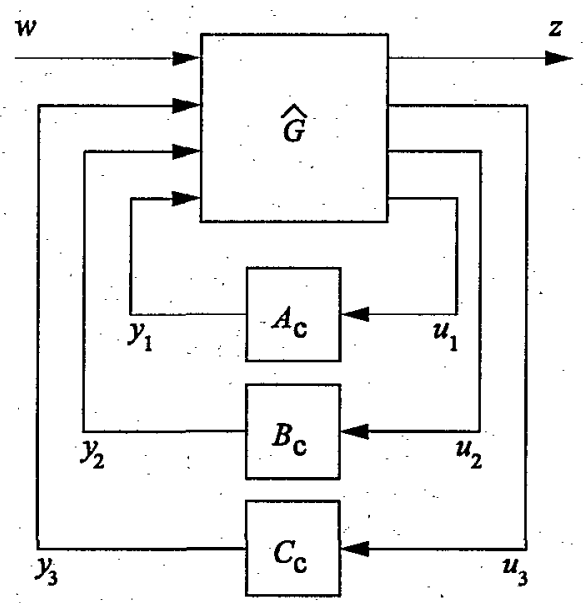

Figure 1: Decentralized Static Output Feedback Framework system shown in Figure 1, where $\hat{G}(s)$ represents the linear, time-invariant dynamic system

$$
\dot{\tilde{x}}(t)=\mathcal{A} \tilde{x}(t)+\sum_{i=1}^{3} \mathcal{B}_{u i} u_{i}(t)+\mathcal{B}_{w} w(t), \quad t \in[0, \infty)(4)
$$

$$
\begin{aligned}
& y_{i}(t)=\mathcal{C}_{y_{i}} \tilde{x}(t)+\mathcal{D}_{y u_{i}} u(t)+\mathcal{D}_{y w_{i}} w(t), \quad i=1,2,3,(5) \\
& z(t)=\mathcal{C}_{z} \tilde{x}(t)+\sum_{i=1}^{3} \mathcal{D}_{z u_{i}} u_{i}(t), \\
& \text { where } \\
& u_{1}(t)=A_{\mathrm{c}} y_{1}(t), \quad u_{2}(t)=B_{\mathrm{c}} y_{2}(t), \quad u_{3}(t)=C_{\mathrm{c}} y_{3}(t), \quad(7)
\end{aligned}
$$

where

and

$$
\begin{aligned}
& \mathcal{A} \triangleq\left[\begin{array}{cc}
A & 0 \\
0 & 0
\end{array}\right], \quad \mathcal{B}_{w} \triangleq\left[\begin{array}{c}
D_{1} \\
0
\end{array}\right], \quad \mathcal{C}_{z} \triangleq\left[\begin{array}{ll}
E_{1} & 0
\end{array}\right] \\
& \mathcal{B}_{u 1} \triangleq\left[\begin{array}{c}
0 \\
I_{n_{\mathrm{c}}}
\end{array}\right], \quad \mathcal{B}_{u 2} \triangleq\left[\begin{array}{c}
0 \\
I_{n_{\mathrm{c}}}
\end{array}\right], \quad \mathcal{B}_{u 3} \triangleq\left[\begin{array}{c}
B \\
0
\end{array}\right] \\
& \mathcal{C}_{y_{1}} \triangleq\left[\begin{array}{ll}
0 & I_{n_{\mathrm{c}}}
\end{array}\right], \quad \mathcal{C}_{y_{2}} \triangleq\left[\begin{array}{ll}
C & 0
\end{array}\right], \quad \mathcal{C}_{y_{3}} \triangleq\left[\begin{array}{lll}
0 & I_{n_{\mathrm{c}}}
\end{array}\right] \\
& \mathcal{D}_{y u_{1}} \triangleq\left[\begin{array}{l}
0 \\
0 \\
0
\end{array}\right], \quad \mathcal{D}_{y_{u_{2}}} \triangleq\left[\begin{array}{l}
0 \\
0 \\
0
\end{array}\right], \quad \mathcal{D}_{y^{u_{3}}} \triangleq\left[\begin{array}{l}
0 \\
D \\
0
\end{array}\right] \\
& \mathcal{D}_{y w_{1}} \triangleq 0, \quad \mathcal{D}_{y w_{2}} \triangleq D_{2}, \quad \mathcal{D}_{y w_{3}} \triangleq 0 \text {, } \\
& \mathcal{D}_{z u 1} \triangleq 0 ; \quad \mathcal{D}_{z u 2} \triangleq 0, \quad \mathcal{D}_{z u 3} \triangleq E_{2} .
\end{aligned}
$$

Next, defining

$$
\hat{u}(t) \triangleq\left[\begin{array}{c}
u_{1}(t) \\
u_{2}(t) \\
u_{3}(t)
\end{array}\right], \quad \hat{y}(t) \triangleq\left[\begin{array}{l}
y_{1}(t) \\
y_{2}(t) \\
y_{3}(t)
\end{array}\right]
$$

$\mathcal{B}_{u} \triangleq\left[\begin{array}{lll}\mathcal{B}_{u 1} & \mathcal{B}_{u 2} & \mathcal{B}_{u 3}\end{array}\right], \quad \mathcal{D}_{z u} \triangleq\left[\begin{array}{lll}\mathcal{D}_{z u 1} & \mathcal{D}_{z u 2} & \mathcal{D}_{z u 3}\end{array}\right]$ $\mathcal{C}_{y} \triangleq\left[\begin{array}{l}\mathcal{C}_{y_{1}} \\ \mathcal{C}_{y_{2}} \\ \mathcal{C}_{y_{3}}\end{array}\right], \mathcal{B}_{u} \triangleq\left[\begin{array}{lll}\mathcal{D}_{y u_{1}} & \mathcal{D}_{y u_{2}} & \mathcal{D}_{y u_{3}}\end{array}\right], \mathcal{D}_{y w} \triangleq\left[\begin{array}{c}\mathcal{D}_{y w_{1}} \\ \mathcal{D}_{y w_{2}} \\ \mathcal{D}_{y w_{3}}\end{array}\right]$

and rewriting the decentralized control signals (7) in the compact form

$$
\hat{u}(t)=\tilde{K} \hat{y}(t)
$$

where

$$
\tilde{K} \triangleq\left[\begin{array}{ccc}
A_{\mathrm{c}} & 0 & 0 \\
0 & B_{\mathrm{c}} & 0 \\
0 & 0 & C_{\mathrm{c}}
\end{array}\right]
$$

the system matrices $\tilde{A}, \tilde{D}$, and $\tilde{E}$ in the closed-loop dynamics

$$
\begin{aligned}
\dot{\tilde{x}}(t) & =\bar{A} \tilde{x}(t)+\bar{D} w(t), \quad t \in[0, \infty) \\
z(t) & =\tilde{E} \tilde{x}(t),
\end{aligned}
$$

can now be written as

$$
\begin{array}{r}
\tilde{A}=\mathcal{A}+\mathcal{B}_{u} \tilde{K} L_{\tilde{K}}{ }^{-1} \mathcal{C}_{y} \\
\tilde{D}=\mathcal{B}_{w}+\mathcal{B}_{u} \tilde{K} L_{\tilde{K}}{ }^{-1} \mathcal{D}_{y w} \\
\tilde{E}=\mathcal{C}_{z}+\mathcal{D}_{z u} \tilde{K} L_{\bar{K}}{ }^{-1} \mathcal{C}_{y}
\end{array}
$$

where

and $\mathcal{D}_{y u}=\left[\begin{array}{lll}\mathcal{D}_{y u_{1}} & \mathcal{D}_{y_{u_{2}}} & \mathcal{D}_{y_{u_{3}}}\end{array}\right]$, 


\section{Fixed-Structure Synthesis for the ACTEX Flight Experiment}

Since continuous-time controllers are implemented on ACTEX, hardware constraints place a limit on the form that the controllers may take. Each of the three struts on ACTEX has a decentralized controller, available in sccond order, third order, fourth order, and fifth order, in the following configurations:

$$
\begin{aligned}
G_{2 c}(s)= & {\left[\frac{k_{3} \omega_{1}^{2}}{s^{2}+.3 \omega_{1} s+\omega_{1}^{2}}+\frac{k_{4} \omega_{2}^{2}}{s^{2}+.3 \omega_{2} s+\omega_{2}^{2}}\right] y } \\
G_{3 c}(s)= & \frac{k_{3} \omega_{2}^{2}}{s^{2}+.3 \omega_{2} s+\omega_{2}^{2}} \times \frac{\omega_{1} s}{s^{2}+.3 \omega_{1} s+\omega_{1}^{2}} y \\
G_{4 c}(s)= & \frac{k_{3} \omega_{2}^{2}}{s^{2}+.3 \omega_{2} s+\omega_{2}^{2}} \times \frac{\omega_{1}^{2}}{s^{2}+.3 \omega_{1} s+\omega_{1}^{2}} y \\
G_{5 c}(s)= & \frac{k_{3} \omega_{3}^{2}}{s^{2}+.3 \omega_{3} s+\omega_{3}^{2}} \\
& \times \frac{\omega_{2}}{s^{2}+.3 \omega_{2} s+\omega_{2}^{2}} \times \frac{\omega_{1} s}{s^{2}+.3 \omega_{1} s+\omega_{1}^{2}} y
\end{aligned}
$$

and where $\omega_{1}, \omega_{2}, \omega_{3}, k_{3}$, and $k_{4}$ are subject to the constraints

$$
0 \leq \omega_{1}, \omega_{2}, \omega_{3} \leq 1024, \quad-16 \leq k_{3} \leq 16, \quad-8 \leq k_{4} \leq 8
$$

Now, we consider the controller associated with strut 1 , and express the controller in the decentralized static output feedback framework. Controller configuration 1, given by (12), in dynamic compensator form, can be expressed as

$$
\begin{gathered}
A_{c}=\left[\begin{array}{cccc}
0 & 1 & 0 & 0 \\
-\omega_{1}^{2} & -0.3 \omega_{1} & 0 & 0 \\
0 & 0 & 0 & 1 \\
0 & 0 & -\omega_{2}^{2} & -0.3 \omega_{2}
\end{array}\right], \quad B_{c}=\left[\begin{array}{l}
0 \\
1 \\
0 \\
1
\end{array}\right], \\
C_{c}=\left[\begin{array}{llll}
k_{3} \omega_{1}^{2} & 0 & k_{4} \omega_{2}^{2} & 0
\end{array}\right] .
\end{gathered}
$$

Controller configurations 2 through 4 , given by (13)-(15), can be expressed similarly, and are given in the Appendix.

Having specified the form of the controller, we consider the $\mathcal{H}_{2}$ synthesis problem. The $\mathcal{H}_{2}$ norm of $G_{z w}(s)$ is given by

$$
\left\|G_{z w}(s)\right\|_{2}^{2}=\operatorname{tr} \tilde{Q} \tilde{R}
$$

where $\tilde{Q}$ is the unique, $\tilde{n} \times \tilde{n}$ nonnegative-definite solution to the algebraic Lyapunov equation

$$
0=\tilde{A} \tilde{Q}+\tilde{Q} \tilde{A}^{\mathrm{T}}+\tilde{D} \tilde{D}^{\mathrm{T}},
$$

where

$$
\tilde{R} \triangleq \tilde{E}^{\mathrm{T}} \tilde{E}=\left[\begin{array}{cc}
R_{1} & 0 \\
0 & C_{\mathrm{c}}^{\mathrm{T}} R_{2} C_{\mathrm{c}}
\end{array}\right]
$$

and $R_{1} \triangleq E_{1}^{\mathrm{T}} E_{1:} R_{2} \triangleq E_{2}^{\mathrm{T}} E_{2}$, and $R_{12} \triangleq E_{1}^{\mathrm{T}} E_{2}=0$.

In order to design $\mathcal{H}_{2}$-optimal controllers for the ACTEX experiment, we pose the following optimization problem: Determine $\tilde{K}$ that minimizes

$$
J(\tilde{K})=\operatorname{tr} \tilde{Q} \tilde{R}
$$

where $\tilde{Q} \geq 0$ satisfies (19). The necessary conditions for optimality can be derived by forming the Lagrangian

$$
\mathcal{L}(\tilde{P}, \tilde{Q}, \tilde{K})=\operatorname{tr} \tilde{Q} \tilde{R}+\operatorname{tr} \tilde{P}\left(\tilde{A} \tilde{Q}+\tilde{Q} \tilde{A}^{\mathrm{T}}+\tilde{V}\right),
$$

where $\tilde{P} \in \mathbb{R}^{\bar{n} \times \bar{n}}$ is a Lagrange multiplier and $\tilde{V}=\tilde{D} \tilde{D}^{\mathrm{T}}$. The partial derivatives with respect to $\tilde{Q}$ and $\tilde{P}$ in (22) are given by

$$
\begin{aligned}
& \frac{\partial \mathcal{L}}{\partial \tilde{Q}}=\tilde{A}^{\mathrm{T}} \tilde{P}+\tilde{P} \tilde{A}+\tilde{R} \\
& \frac{\partial \mathcal{L}}{\partial \tilde{P}}=\tilde{A} \tilde{Q}+\tilde{Q} \tilde{A}^{\mathrm{T}}+\tilde{V}
\end{aligned}
$$

To obtain the partial derivative of the Lagrangian with respect to the free parameters in the controller gains, we first specify the controller configuration. As an example, we consider controller configuration $1,(12)$. The settings for each of the other controller configurations are given in the Appendix.

For controller configuration 1, with (17), the block-diagonal matrix $\tilde{K}$ has the form (9). Note that this controller has four free optimization parameters, namely, $\omega_{1}, \omega_{2}, k_{3}$, and $k_{4}$. We construct the matrix

$$
K=\left[\begin{array}{cccc}
\omega_{1} & 0 & 0 & 0 \\
0 & \omega_{2} & 0 & 0 \\
0 & 0 & k_{3} & 0 \\
0 & 0 & 0 & k_{4}
\end{array}\right]
$$

and note that

$$
\tilde{K}=K_{0}+L_{1} K R_{1}+L_{2} K K R_{2}+L_{3} K K M_{3} K R_{3},
$$

where

$$
\begin{aligned}
& K_{0}=\left[\begin{array}{lllllllll}
0 & 1 & 0 & 0 & 0 & 0 & 0 & 0 & 0 \\
0 & 0 & 0 & 0 & 0 & 0 & 0 & 0 & 0 \\
0 & 0 & 0 & 1 & 0 & 0 & 0 & 0 & 0 \\
0 & 0 & 0 & 0 & 0 & 0 & 0 & 0 & 0 \\
0 & 0 & 0 & 0 & 0 & 0 & 0 & 0 & 0 \\
0 & 0 & 0 & 0 & 1 & 0 & 0 & 0 & 0 \\
0 & 0 & 0 & 0 & 0 & 0 & 0 & 0 & 0 \\
0 & 0 & 0 & 0 & 1 & 0 & 0 & 0 & 0 \\
0 & 0 & 0 & 0 & 0 & 0 & 0 & 0 & 0
\end{array}\right], \\
& L_{1}=\left[\begin{array}{cccc}
0 & 0 & 0 & 0 \\
-0.3 & 0 & 0 & 0 \\
0 & 0 & 0 & 0 \\
0 & -0.3 & 0 & 0 \\
0 & 0 & 0 & 0 \\
0 & 0 & 0 & 0 \\
0 & 0 & 0 & 0 \\
0 & 0 & 0 & 0 \\
0 & 0 & 0 & 0
\end{array}\right], R_{1}=\left[\begin{array}{lllll}
0 & 0 & 0 & 0 \\
1 & 0 & 0 & 0 \\
0 & 0 & 0 & 0 \\
0 & 1 & 0 & 0 \\
0 & 0 & 0 & 0 \\
0 & 0 & 0 & 0 \\
0 & 0 & 0 & 0 \\
0 & 0 & 0 & 0 \\
0 & 0 & 0 & 0
\end{array}\right]^{\mathrm{T}},
\end{aligned}
$$




$$
\begin{aligned}
& L_{2}=\left[\begin{array}{cccc}
0 & 0 & 0 & 0 \\
-1 & 0 & 0 & 0 \\
0 & 0 & 0 & 0 \\
0 & -1 & 0 & 0 \\
0 & 0 & 0 & 0 \\
0 & 0 & 0 & 0 \\
0 & 0 & 0 & 0 \\
0 & 0 & 0 & 0 \\
0 & 0 & 0 & 0
\end{array}\right], \quad R_{2}=\left[\begin{array}{llll}
1 & 0 & 0 & 0 \\
0 & 0 & 0 & 0 \\
0 & 1 & 0 & 0 \\
0 & 0 & 0 & 0 \\
0 & 0 & 0 & 0 \\
0 & 0 & 0 & 0 \\
0 & 0 & 0 & 0 \\
0 & 0 & 0 & 0 \\
0 & 0 & 0 & 0
\end{array}\right]^{\mathrm{T}} \\
& L_{3}=\left[\begin{array}{llll}
0 & 0 & 0 & 0 \\
0 & 0 & 0 & 0 \\
0 & 0 & 0 & 0 \\
0 & 0 & 0 & 0 \\
0 & 0 & 0 & 0 \\
0 & 0 & 0 & 0 \\
0 & 0 & 0 & 0 \\
0 & 0 & 0 & 0 \\
1 & 1 & 0 & 0
\end{array}\right], M_{3}=\left[\begin{array}{llll}
0 & 0 & 1 & 0 \\
0 & 0 & 0 & 1 \\
0 & 0 & 0 & 0 \\
0 & 0 & 0 & 0
\end{array}\right], \\
& R_{3}=\left[\begin{array}{lllllllll}
0 & 0 & 0 & 0 & 0 & 0 & 0 & 0 & 0 \\
0 & 0 & 0 & 0 & 0 & 0 & 0 & 0 & 0 \\
0 & 0 & 0 & 0 & 0 & 1 & 0 & 0 & 0 \\
0 & 0 & 0 & 0 & 0 & 0 & 0 & 1 & 0
\end{array}\right] \text {. }
\end{aligned}
$$

Now with $\tilde{K}$ in terms of $K$, we can take the derivative of the Lagrangian with respect to $K$. Define the following notation

$$
\begin{gathered}
\tau \triangleq I+\tilde{K} L_{\bar{K}}{ }^{-1} \mathcal{D}_{y u}, \quad \hat{A} \triangleq \tau^{\mathrm{T}} \mathcal{B}_{u}^{\mathrm{T}} \tilde{P} \tilde{Q} \mathcal{C}_{y}^{\mathrm{T}} L_{\bar{K}}{ }^{-\mathrm{T}}, \\
\hat{D} \triangleq \tau^{\mathrm{T}} \mathcal{B}_{u}^{\mathrm{T}} \tilde{P} \tilde{D} \mathcal{D}_{y w}^{\mathrm{T}} L_{\bar{K}}{ }^{-\mathrm{T}}, \quad \hat{E} \triangleq \tau^{\mathrm{T}} \mathcal{D}_{z u}^{\mathbf{T}} \tilde{E} \tilde{Q} \mathcal{C}_{y}^{\mathbf{T}} L_{\tilde{K}}{ }^{-\mathrm{T}} .
\end{gathered}
$$

The derivative of the Lagrangian with respect to $K$, for each controller configuration, is given by

$$
\begin{aligned}
\frac{1}{2} \frac{\partial C}{\partial K}= & L_{1}^{\mathrm{T}} \hat{A} R_{1}^{\mathrm{T}}+L_{2}^{\mathrm{T}} \hat{A} R_{2}^{\mathrm{T}} K+K L_{2}^{\mathrm{T}} \hat{A} R_{2}^{\mathrm{T}}+L_{3}^{\mathrm{T}} \hat{A} R_{3}^{\mathrm{T}} K M_{3}^{\mathrm{T}} K \\
& +K L_{3}^{\mathrm{T}} \hat{A} R_{3}^{\mathrm{T}} K M_{3}^{\mathrm{T}}+M_{3}^{\mathrm{T}} K K L_{3}^{\mathrm{T}} \hat{A} R_{3}^{\mathrm{T}}+L_{1}^{\mathrm{T}} \hat{D} R_{1}^{\mathrm{T}} \\
& +L_{2}^{\mathrm{T}} \hat{D} R_{2}^{\mathrm{T}} K+K L_{2}^{\mathbf{T}} \hat{D} R_{2}^{\mathrm{T}}+L_{3}^{\mathrm{T}} \hat{D} R_{3}^{\mathrm{T}} K M_{3}^{\mathrm{T}} K \\
& +K L_{3}^{\mathrm{T}} \hat{D} R_{3}^{\mathrm{T}} K M_{3}^{\mathrm{T}}+M_{3}^{\mathrm{T}} K K L_{3}^{\mathrm{T}} \hat{D} R_{3}^{\mathrm{T}}+L_{1}^{\mathrm{T}} \hat{E} R_{1}^{\mathrm{T}} \\
& +L_{2}^{\mathrm{T}} \hat{E} R_{2}^{\mathrm{T}} K+K L_{2}^{\mathrm{T}} \hat{E} R_{2}^{\mathrm{T}}+L_{3}^{\mathrm{T}} \hat{E} R_{3}^{\mathrm{T}} K M_{3}^{\mathrm{T}} K \\
& +K L_{3}^{\mathrm{T}} \hat{E} R_{3}^{\mathrm{T}} K M_{3}^{\mathrm{T}}+M_{3}^{\mathrm{T}} K K L_{3}^{\mathrm{T}} \hat{E} R_{3}^{\mathrm{T}}
\end{aligned}
$$

\section{Quasi-Newton Algorithm}

To solve the nonlinear optimization problem posed in Section, a general-purpose BFGS quasi-Newton algorithm [9] is used. The line-search portions of the algorithm were modified to include a constraint-checking subroutine to verify that the search direction vector lies entirely within the set of parameters that yield a stable closed-loop system as well as lying within the allowable limits of the free parameters given by (16). This modification ensures that the cost function $J$ remains defined at every point in the line-search process.

One requirement of gradient-based optimization algorithms is an initial stabilizing design. Initial designs showed that for large values of $\omega_{i}, i-1,2,3$, the cost function depended very weakly upon these values. Therefore, $\omega_{i}, i=1,2,3$, were initially chosen to be 48,72 , and 96 , respectively. Furthermore, since the open-loop system was stable, an initial stabilizing design could be obtained by setting $k_{3}=k_{4}=0$. With these values set: the BFGS quasi-Newton algorithm was applied to find the $\mathcal{H}_{2}$-optimal solution for a given fixedstructure controller configuration.

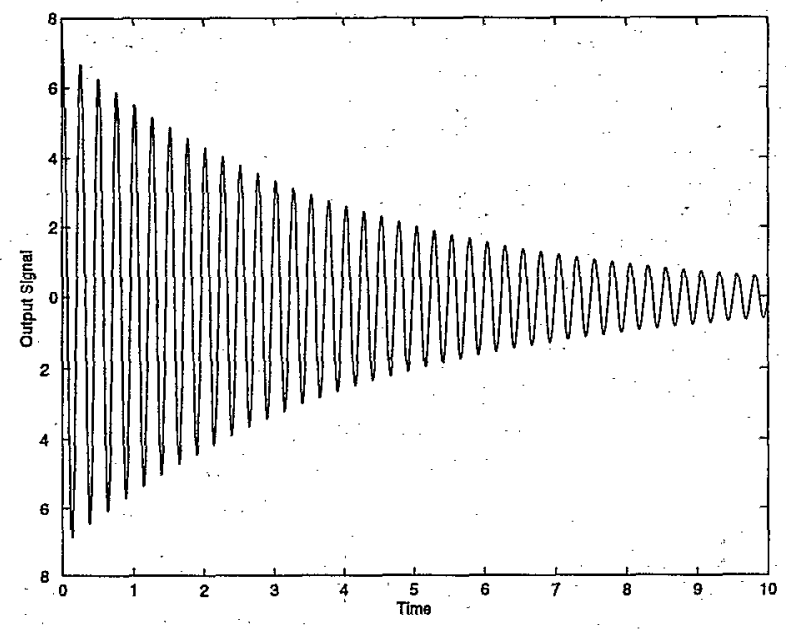

Figure 2: Open-Loop Impulse Response

\section{Simulation Results}

The ACTEX experiment is a lightly damped flexible structure, as can be seen by the impulse response shown in Figure 2, where

$$
x(0)=\left[\begin{array}{cccc}
-0 & 0 & 0 & 1
\end{array}\right]^{\mathrm{T}} .
$$

For each of the four different controller configurations, three different controllers were designed by setting the control weighting matrix $R_{2}$ to $1,0.01$, or 0.0001 . The $\mathcal{H}_{2}$ cost of these controllers can be seen in Table 1. It is seen that the $\mathcal{H}_{2}$ cost is generally best for the first controller configuration. In fact, the other controller configurations ran into stability constraints, as explained in Section. Therefore, the optimization routine terminated due to a boundary

\begin{tabular}{|l||c|c|c|c|}
\hline & Config. 1 & Config. 2 & Config. 3 & Config. 4 \\
\hline \hline$R_{2}=1$ & 0.6457 & 0.7076 & 0.5833 & 0.7216 \\
\hline$R_{2}=0.01$ & 0.2444 & 0.5012 & 0.6264 & 0.3769 \\
\hline$R_{2}=0.0001$ & 0.1910 & 0.5942 & 0.6246 & 0.5618 \\
\hline
\end{tabular}

Table 1: $\mathcal{H}_{2}$ Costs for Various Controller Configurations and Weightings 


\begin{tabular}{|l||c|c|c|c|}
\hline & $\omega_{1}$ & $\omega_{2}$ & $k_{3}$ & $k_{4}$ \\
\hline \hline$R_{2}=1$ & 31.081 & 70.702 & 0.184 & -0.125 \\
\hline$R_{2}=0.01$ & 36.443 & 99.118 & 0.320 & 0.402 \\
\hline$R_{2}=0.0001$ & 37.366 & 123.840 & 0.208 & 0.631 \\
\hline
\end{tabular}

Table 2: Optimal Controller Parameters for Controller Configuration 1

constraint rather than a small gradient condition. However, the optimization routine worked well for the first controller configuration, and it can be seen from Table 1 that the $\mathcal{H}_{2}$ cost of the closed-loop system decreases with increasing controller authority.

The optimal controller parameters for this controller configuration are given in Table 2 . In fact, with $R_{2}=1$, we can see from the output signals of the impulse response in Figure 3 that this controller does not attenuate the vibrations significantly. However, as the authority is increased, the attenuation becomes greater, as seen in Figure 4 and Figure 5, though this does increase the control effort expended, as shown in Figures 6-8, which could lead to actuator saturation.

Finally, Figure 9 shows the Bode plots of the open-loop and closed-loop systems. It is seen that the size of the first peak in the closed-loop response is decreased as the controller authority is increased. Another important feature of the closed-loop frequency response is the high-frequency rolloff. Since the ACTEX system model has a relative degree of zero, the system possesses gain at all frequencies, thus unmodeled high-frequency dynamics could destabilize the open-loop system, whereas these dynamics would be attenuated with a strictly proper dynamic controller.

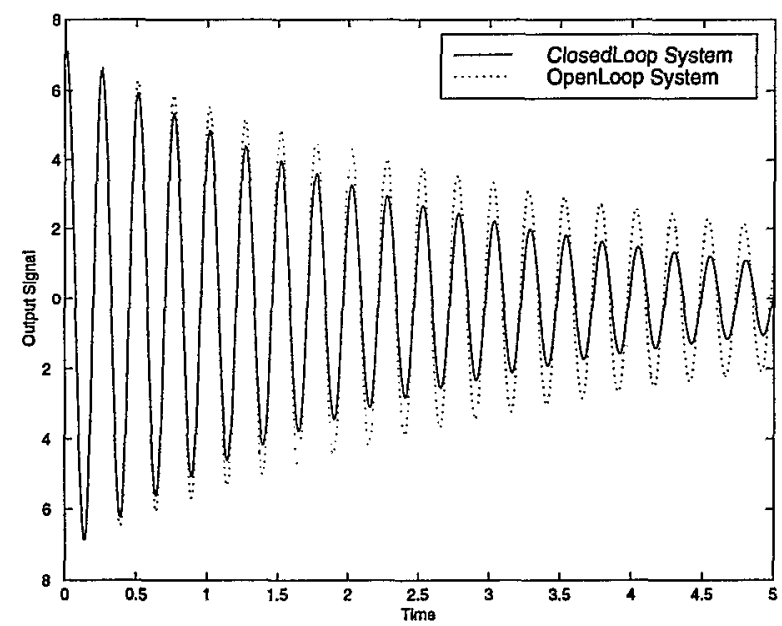

Figure 3: Closed-Loop Impulse Response, $R_{2}=1$

\section{Conclusions}

In this paper we showed that the control algorithm structure implemented on the ACTEX flight experiment has the form of a decentralized static output feedback controller. This controller structure is amenable to fixed-structure optimization methods. Using the Robust, Fixed-Structure Toolbox, we synthesized several feedback controllers and we assessed their performance under a variety of conditions.

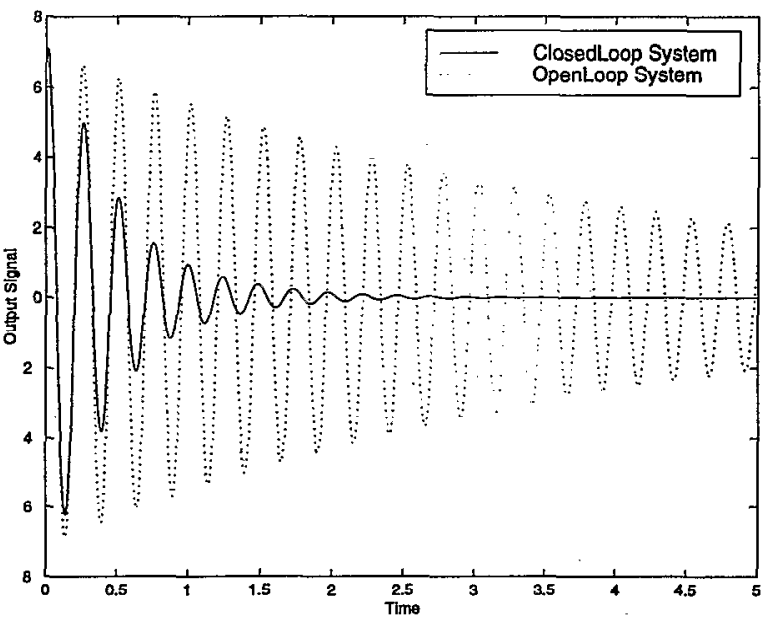

Figurc 4: Closed-Loop Impulse Response, $R_{2}=0.01$

\section{References}

[1] D. C. Hyland and D. S. Bernstein, "The Optimal Pro jection Equations for Fixed-Order Dynamic Compensation," IEEE Trans. Autom. Contr., Vol. AC-29, pp. 1034-1037, 1984.

[2] D. S. Bernstein and W. M. Haddad, "LQG Control With an $\mathrm{H}_{\infty}$ Performance Bound: A Riccati Equation Approach," IEEE Trans. Autom. Contr., Vol. 34, pp. 293-305, 1989.

[3] D. S. Bernstein and W. M. Haddad, "Robust Stability and Performance via Fixed-Order Dynamic Compensation with Guaranteed Cost Bounds," Math. Contr. Sig. Sys., Vol. 3, pp. 139-163, 1990.

[4] W. M. Haddad and D. S. Bernstein, "Generalized Riccati Equations for the Full- and Reduced-Order MixedNorm $\mathrm{H}_{2} / \mathrm{H}_{\infty}$ Standard Problem," Sys. Contr. Lett., Vol. 14, pp. 185-197, 1990.

[5] Y. Ge, L. T. Watson, E. G. Collins, Jr., and D. S. Bernstein, "Probability-One Homotopy Algorithms for 


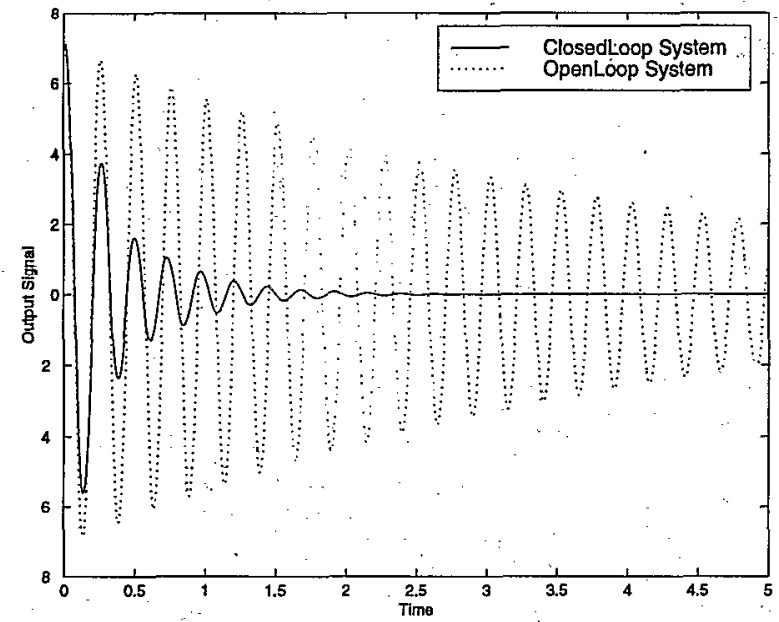

Figure 5: Closed-Loop Impulse Response, $R_{2}=0.0001$

Full and Reduced Order $\mathrm{H}^{2} / \mathrm{H}^{\infty}$ Controller Synthesis," Optimal Contr. Appl. Meth., Vol, 17, pp. 187-208, 1996.

[6] R. S. Erwin, A. G. Sparks, and D. S. Bernstein, "FixedStructure Robust Controller Synthesis via Decentralized Static Output Feedback," Int. J. Robust Nonlinear Contr., Vol. 8, pp. 499-522, 1998.

[7] R. S. Erwin and D. S. Bernstein, "Fixed-Structure Discrete-Time Mixed $\mathrm{H}_{2} / \mathrm{H}_{\infty}$ Controller Synthesis Using the Delta-Operator," submitted to Int. J. Contr.

[8] R. S. Erwin, D. S. Bernstein, and D. A. Wilson, "FixedStructure Synthesis of Induced-Norm Controllers," submitted to Int. J. Contr.

[9] J. E. Dennis, Jr. and R. B. Schnabel. Numerical Methods for Unconstrained Optimization and Nonlinear Equations. Prentice-Hall, 1983.

\section{Appendix}

\section{Controller Configuration 2}

For this contiguration, the controller given by (13) can be expressed as

$$
\begin{aligned}
A_{\mathrm{c}} & =\left[\begin{array}{cccc}
0 & 1 & 0 & 0 \\
-\omega_{1}^{2} & -0.3 \omega_{1} & 0 & 0 \\
0 & 0 & 0 & 1 \\
0 & \omega_{1} & -\omega_{2}^{2} & -0.3 \omega_{2}
\end{array}\right], \\
B_{\mathrm{c}} & =\left[\begin{array}{l}
0 \\
1 \\
0 \\
0
\end{array}\right], \quad C_{\mathrm{c}}=\left[\begin{array}{llll}
0 & 0 & k_{3} \omega_{2}^{2} & 0
\end{array}\right] .
\end{aligned}
$$

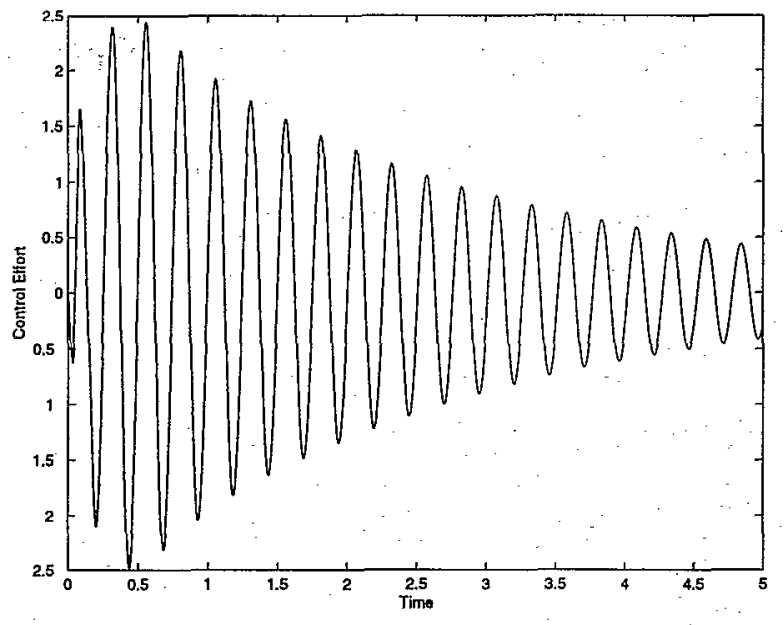

Figure 6: Closed-Loop Control Effort, $R_{2}=1$

Note that there are three free parameters, namely, $\omega_{1}, \omega_{2}$, and $k_{3}$. Thus

$$
K=\left[\begin{array}{ccc}
\omega_{1} & 0 & 0 \\
0 & \omega_{2} & 0 \\
0 & 0 & k_{3}
\end{array}\right]
$$

and

$$
K_{0}=\left[\begin{array}{lllllllll}
0 & 1 & 0 & 0 & 0 & 0 & 0 & 0 & 0 \\
0 & 0 & 0 & 0 & 0 & 0 & 0 & 0 & 0 \\
0 & 0 & 0 & 1 & 0 & 0 & 0 & 0 & 0 \\
0 & 0 & 0 & 0 & 0 & 0 & 0 & 0 & 0 \\
0 & 0 & 0 & 0 & 0 & 0 & 0 & 0 & 0 \\
0 & 0 & 0 & 0 & 1 & 0 & 0 & 0 & 0 \\
0 & 0 & 0 & 0 & 0 & 0 & 0 & 0 & 0 \\
0 & 0 & 0 & 0 & 0 & 0 & 0 & 0 & 0 \\
0 & 0 & 0 & 0 & 0 & 0 & 0 & 0 & 0
\end{array}\right]
$$$$
L_{1}=\left[\begin{array}{ccc}
0 & 0 & 0 \\
-0.3 & 0 & 0 \\
0 & 0 & 0 \\
1 & -0.3 & 0 \\
0 & 0 & 0 \\
0 & 0 & 0 \\
0 & 0 & 0 \\
0 & 0 & 0 \\
0 & 0 & 0
\end{array}\right], \quad R_{1}=\left[\begin{array}{lll}
0 & 0 & 0 \\
1 & 0 & 0 \\
0 & 0 & 0 \\
0 & 1 & 0 \\
0 & 0 & 0 \\
0 & 0 & 0 \\
0 & 0 & 0 \\
0 & 0 & 0 \\
0 & 0 & 0
\end{array}\right]^{\mathrm{T}}
$$

$$
L_{2}=\left[\begin{array}{ccc}
0 & 0 & 0 \\
-1 & 0 & 0 \\
0 & 0 & 0 \\
0 & -1 & 0 \\
0 & 0 & 0 \\
0 & 0 & 0 \\
0 & 0 & 0 \\
0 & 0 & 0 \\
0 & 0 & 0
\end{array}\right], R_{2}=\left[\begin{array}{ccccccccc}
1 & 0 & 0 & 0 & 0 & 0 & 0 & 0 & 0 \\
0 & 0 & 1 & 0 & 0 & 0 & 0 & 0 & 0 \\
0 & 0 & 0 & 0 & 0 & 0 & 0 & 0 & 0
\end{array}\right]
$$




$$
\begin{aligned}
L_{3} & =\left[\begin{array}{lll}
0 & 0 & 0 \\
0 & 0 & 0 \\
0 & 0 & 0 \\
0 & 0 & 0 \\
0 & 0 & 0 \\
0 & 0 & 0 \\
0 & 0 & 0 \\
0 & 0 & 0 \\
0 & 1 & 0
\end{array}\right], M_{3}=\left[\begin{array}{lll}
0 & 0 & 0 \\
0 & 0 & 1 \\
0 & 0 & 0
\end{array}\right] \\
R_{3} & =\left[\begin{array}{lllllllll}
0 & 0 & 0 & 0 & 0 & 0 & 0 & 0 & 0 \\
0 & 0 & 0 & 0 & 0 & 0 & 0 & 0 & 0 \\
0 & 0 & 0 & 0 & 0 & 0 & 0 & 1 & 0
\end{array}\right]
\end{aligned}
$$

\section{Controller Configuration 3}

For this configuration, the controller given by (14) can be expressed as

$$
\begin{aligned}
& A_{\mathrm{c}}=\left[\begin{array}{cccc}
0 & 1 & 0 & 0 \\
-\omega_{1}^{2} & -0.3 \omega_{1} & 0 & 0 \\
0 & 0 & 0 & 1 \\
\omega_{1}^{2} & 0 & -\omega_{2}^{2} & -0.3 \omega_{2}
\end{array}\right] \\
& B_{\mathrm{c}}=\left[\begin{array}{l}
0 \\
1 \\
0 \\
0
\end{array}\right], \quad C_{\mathrm{c}}=\left[\begin{array}{llll}
0 & 0 & k_{3} \omega_{2}^{2} & 0
\end{array}\right] .
\end{aligned}
$$

Note that there are three free parameters, namely, $\omega_{1}, \omega_{2}$, and $k_{3}$. Thus

$$
K=\left[\begin{array}{ccc}
\omega_{1} & 0 & 0 \\
0 & \omega_{2} & 0 \\
0 & 0 & k_{3}
\end{array}\right]
$$

and

$$
\begin{gathered}
K_{0}=\left[\begin{array}{lllllllll}
0 & 1 & 0 & 0 & 0 & 0 & 0 & 0 & 0 \\
0 & 0 & 0 & 0 & 0 & 0 & 0 & 0 & 0 \\
0 & 0 & 0 & 1 & 0 & 0 & 0 & 0 & 0 \\
0 & 0 & 0 & 0 & 0 & 0 & 0 & 0 & 0 \\
0 & 0 & 0 & 0 & 0 & 0 & 0 & 0 & 0 \\
0 & 0 & 0 & 0 & 1 & 0 & 0 & 0 & 0 \\
0 & 0 & 0 & 0 & 0 & 0 & 0 & 0 & 0 \\
0 & 0 & 0 & 0 & 0 & 0 & 0 & 0 & 0 \\
0 & 0 & 0 & 0 & 0 & 0 & 0 & 0 & 0
\end{array}\right], \\
L_{1}=\left[\begin{array}{ccc}
0 & 0 & 0 \\
-0.3 & 0 & 0 \\
0 & 0 & 0 \\
1 & -0.3 & 0 \\
0 & 0 & 0 \\
0 & 0 & 0 \\
0 & 0 & 0 \\
0 & 0 & 0 \\
0 & 0 & 0
\end{array}\right], R_{1}=\left[\begin{array}{llll}
0 & 0 & 0 \\
1 & 0 & 0 \\
0 & 0 & 0 \\
0 & 1 & 0 \\
0 & 0 & 0 \\
0 & 0 & 0 \\
0 & 0 & 0 \\
0 & 0 & 0 \\
0 & 0 & 0
\end{array}\right]^{\mathrm{T}}
\end{gathered}
$$$$
L_{2}=\left[\begin{array}{ccc}
0 & 0 & 0 \\
-1 & 0 & 0 \\
0 & 0 & 0 \\
1 & -1 & 0 \\
0 & 0 & 0 \\
0 & 0 & 0 \\
0 & 0 & 0 \\
0 & 0 & 0 \\
0 & 0 & 0
\end{array}\right], R_{2}=\left[\begin{array}{ccccccccc}
1 & 0 & 0 & 0 & 0 & 0 & 0 & 0 & 0 \\
0 & 0 & 1 & 0 & 0 & 0 & 0 & 0 & 0 \\
0 & 0 & 0 & 0 & 0 & 0 & 0 & 0 & 0
\end{array}\right],
$$$$
L_{3}=\left[\begin{array}{lll}
0 & 0 & 0 \\
0 & 0 & 0 \\
0 & 0 & 0 \\
0 & 0 & 0 \\
0 & 0 & 0 \\
0 & 0 & 0 \\
0 & 0 & 0 \\
0 & 0 & 0 \\
0 & 1 & 0
\end{array}\right], \quad M_{3}=\left[\begin{array}{lll}
0 & 0 & 0 \\
0 & 0 & 1 \\
0 & 0 & 0
\end{array}\right]
$$$$
R_{3}=\left[\begin{array}{lllllllll}
0 & 0 & 0 & 0 & 0 & 0 & 0 & 0 & 0 \\
0 & 0 & 0 & 0 & 0 & 0 & 0 & 0 & 0 \\
0 & 0 & 0 & 0 & 0 & 0 & 0 & 1 & 0
\end{array}\right]
$$

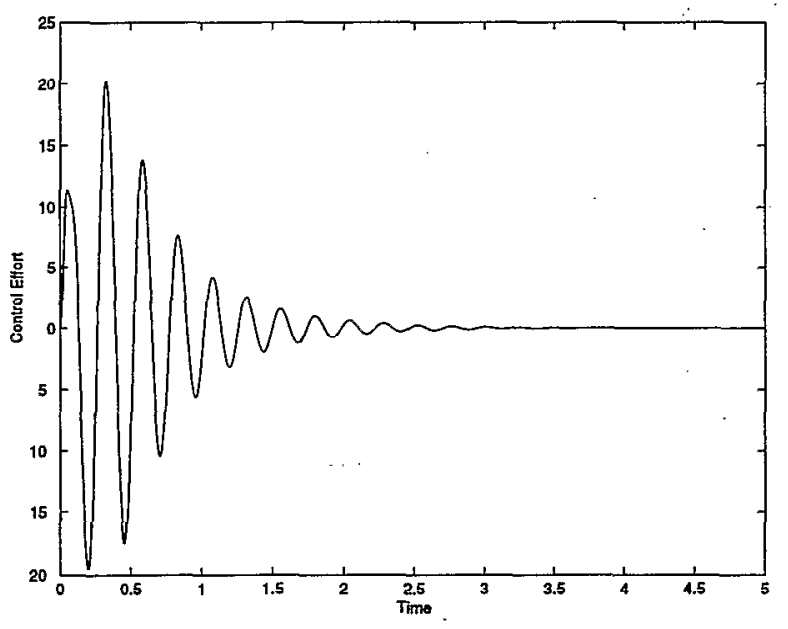

Figure 7: Closed-Loop Control Effort, $R_{2}=0.01$ 


\section{Controller Configuration 4}

For this configuration, the controller given by (15) can be expressed as

$$
\begin{gathered}
A_{\mathrm{c}}=\left[\begin{array}{cccccc}
0 & 1 & 0 & 0 & 0 & 0 \\
-\omega_{1}^{2} & -0.3 \omega_{1} & 0 & 0 & 0 & 0 \\
0 & 0 & 0 & 1 & 0 & 0 \\
0 & \omega_{1} & -\omega_{2}^{2} & -0.3 \omega_{2} & 0 & 0 \\
0 & 0 & 0 & 0 & 0 & 1 \\
0 & 0 & \omega_{2}^{2} & 0 & -\omega_{3}^{2} & -0.3 \omega_{3}
\end{array}\right] \\
B_{\mathbf{c}}=\left[\begin{array}{l}
0 \\
1 \\
0 \\
0 \\
0 \\
0
\end{array}\right], \quad C_{\mathrm{c}}=\left[\begin{array}{llllll}
0 & 0 & 0 & 0 & k_{3} \omega_{3}^{2} & 0
\end{array}\right]
\end{gathered}
$$

Note that there are four free parameters, namely, $\omega_{1}, \omega_{2}$, $\omega_{3}$, and $k_{3}$. Thus

$$
K=\left[\begin{array}{cccc}
\omega_{1} & 0 & 0 & 0 \\
0 & \omega_{2} & 0 & 0 \\
0 & 0 & \omega_{3} & 0 \\
0 & 0 & 0 & k_{3}
\end{array}\right]
$$

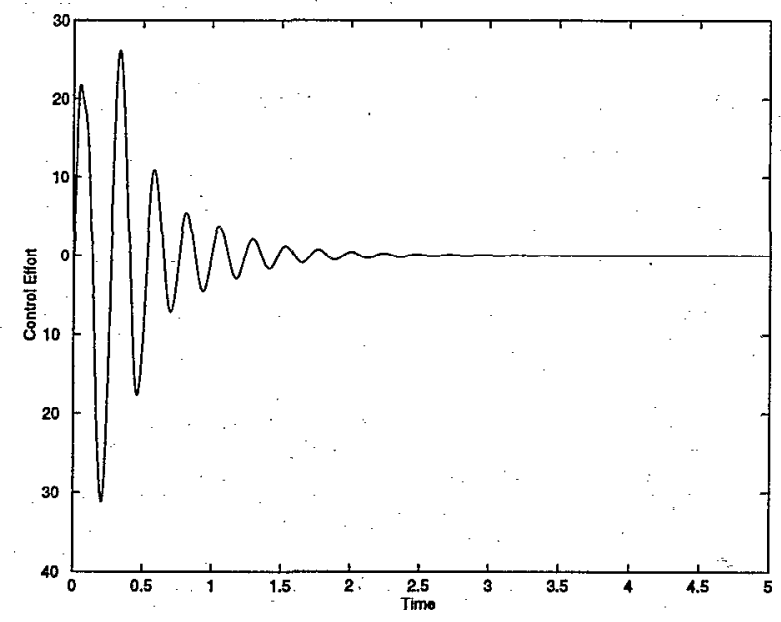

Figure 8: Closed-Loop Control Effort, $R_{2}=0.0001$
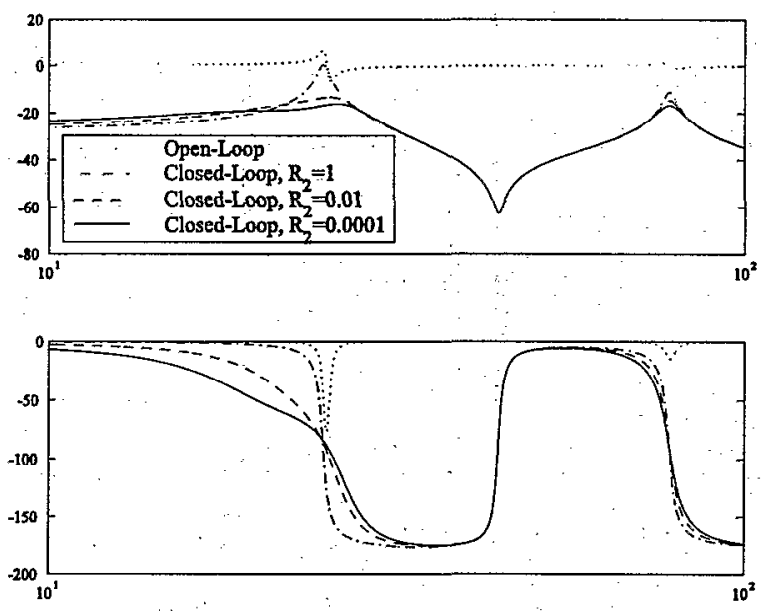

Figure 9: Bode Plots for Open-Loop and Closed-Loop Systems

and

$$
\begin{aligned}
& {\left[\begin{array}{lllllllllllll}
0 & 1 & 0 & 0 & 0 & 0 & 0 & 0 & 0 & 0 & 0 & 0 & 0 \\
0 & 0 & 0 & 0 & 0 & 0 & 0 & 0 & 0 & 0 & 0 & 0 & 0 \\
0 & 0 & 0 & 1 & 0 & 0 & 0 & 0 & 0 & 0 & 0 & 0 & 0 \\
0 & 0 & 0 & 0 & 0 & 0 & 0 & 0 & 0 & 0 & 0 & 0 & 0 \\
0 & 0 & 0 & 0 & 0 & 1 & 0 & 0 & 0 & 0 & 0 & 0 & 0 \\
0 & 0 & 0 & 0 & 0 & 0 & 0 & 0 & 0 & 0 & 0 & 0 & 0 \\
0 & 0 & 0 & 0 & 0 & 0 & 0 & 0 & 0 & 0 & 0 & 0 & 0 \\
0 & 0 & 0 & 0 & 0 & 0 & 1 & 0 & 0 & 0 & 0 & 0 & 0 \\
0 & 0 & 0 & 0 & 0 & 0 & 0 & 0 & 0 & 0 & 0 & 0 & 0 \\
0 & 0 & 0 & 0 & 0 & 0 & 0 & 0 & 0 & 0 & 0 & 0 & 0 \\
0 & 0 & 0 & 0 & 0 & 0 & 0 & 0 & 0 & 0 & 0 & 0 & 0 \\
0 & 0 & 0 & 0 & 0 & 0 & 0 & 0 & 0 & 0 & 0 & 0 & 0 \\
0 & 0 & 0 & 0 & 0 & 0 & 0 & 0 & 0 & 0 & 0 & 0 & 0
\end{array}\right]} \\
& L_{1}=\left[\begin{array}{cccc}
0 & 0 & 0 & 0 \\
-0.3 & 0 & 0 & 0 \\
0 & 0 & 0 & 0 \\
1 & -0.3 & 0 & 0 \\
0 & 0 & 0 & 0 \\
0 & 0 & -0.3 & 0 \\
0 & 0 & 0 & 0 \\
0 & 0 & 0 & 0 \\
0 & 0 & 0 & 0 \\
0 & 0 & 0 & 0 \\
0 & 0 & 0 & 0 \\
0 & 0 & 0 & 0 \\
0 & 0 & 0 & 0
\end{array}\right] ; \quad R_{1}=\left[\begin{array}{llll}
0 & 0 & 0 & 0 \\
1 & 0 & 0 & 0 \\
0 & 0 & 0 & 0 \\
0 & 1 & 0 & 0 \\
0 & 0 & 0 & 0 \\
0 & 0 & 1 & 0 \\
0 & 0 & 0 & 0 \\
0 & 0 & 0 & 0 \\
0 & 0 & 0 & 0
\end{array}\right]^{\mathrm{T}}
\end{aligned}
$$




$$
L_{2}=\left[\begin{array}{cccc}
0 & 0 & 0 & 0 \\
-1 & 0 & 0 & 0 \\
0 & 0 & 0 & 0 \\
0 & -1 & 0 & 0 \\
0 & 0 & 0 & 0 \\
0 & 1 & -1 & 0 \\
0 & 0 & 0 & 0 \\
0 & 0 & 0 & 0 \\
0 & 0 & 0 & 0 \\
0 & 0 & 0 & 0 \\
0 & 0 & 0 & 0 \\
0 & 0 & 0 & 0 \\
0 & 0 & 0 & 0
\end{array}\right]
$$

$$
R_{2}=\left[\begin{array}{lllllllllllll}
1 & 0 & 0 & 0 & 0 & 0 & 0 & 0 & 0 & 0 & 0 & 0 & 0 \\
0 & 0 & 1 & 0 & 0 & 0 & 0 & 0 & 0 & 0 & 0 & 0 & 0 \\
0 & 0 & 0 & 0 & 1 & 0 & 0 & 0 & 0 & 0 & 0 & 0 & 0 \\
0 & 0 & 0 & 0 & 0 & 0 & 0 & 0 & 0 & 0 & 0 & 0 & 0
\end{array}\right]
$$$$
L_{3}=\left[\begin{array}{llll}
0 & 0 & 0 & 0 \\
0 & 0 & 0 & 0 \\
0 & 0 & 0 & 0 \\
0 & 0 & 0 & 0 \\
0 & 0 & 0 & 0 \\
0 & 0 & 0 & 0 \\
0 & 0 & 0 & 0 \\
0 & 0 & 0 & 0 \\
0 & 0 & 0 & 0 \\
0 & 0 & 0 & 0 \\
0 & 0 & 0 & 0 \\
0 & 0 & 0 & 0 \\
0 & 0 & 1 & 0
\end{array}\right], \quad M_{3}=\left[\begin{array}{llll}
0 & 0 & 0 & 0 \\
0 & 0 & 0 & 0 \\
0 & 0 & 0 & 1 \\
0 & 0 & 0 & 0
\end{array}\right] \text {, }
$$$$
R_{3}=\left[\begin{array}{lllllllllllll}
0 & 0 & 0 & 0 & 0 & 0 & 0 & 0 & 0 & 0 & 0 & 0 & 0 \\
0 & 0 & 0 & 0 & 0 & 0 & 0 & 0 & 0 & 0 & 0 & 0 & 0 \\
0 & 0 & 0 & 0 & 0 & 0 & 0 & 0 & 0 & 0 & 0 & 0 & 0 \\
0 & 0 & 0 & 0 & 0 & 0 & 0 & 0 & 0 & 0 & 0 & 1 & 0
\end{array}\right]
$$ 